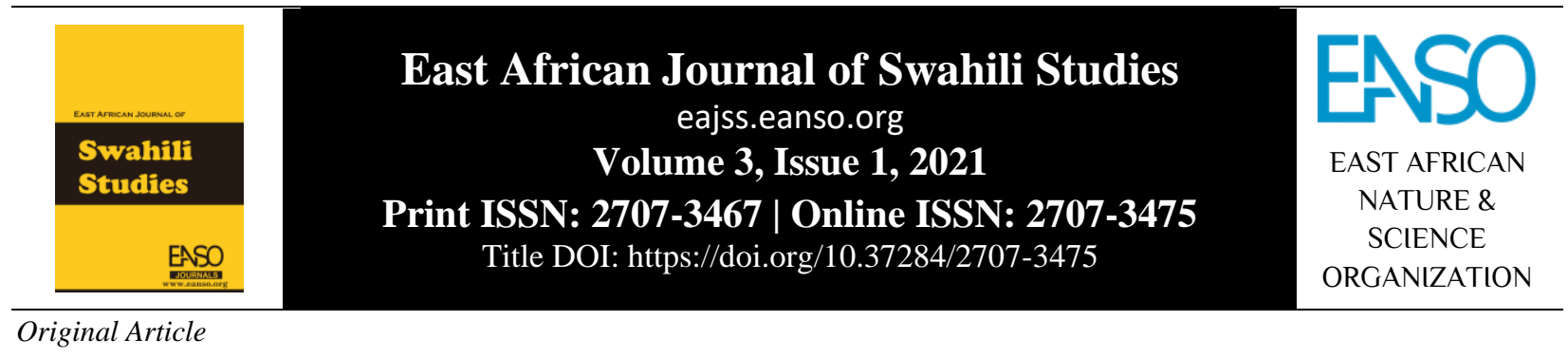

\title{
Mchango wa Tanzu za Kimaigizo za Fasihi Simulizi Katika Umahuluti wa Riwaya ya Kiswahili: Uchunguzi wa Riwaya ya Kufa Kuzikana
}

\author{
Simiyu Benson Sululu ${ }^{I}$, Dkt. Richard Makhanu Wafula, PhD ${ }^{I}$ na Dkt. Joseph Nyehita Maitaria, PhD \\ ${ }^{1}$ Mwanafunzi wa Uzamifu, Chuo Kikuu cha Kenyatta, S.L.P 126- 50202, Nairobi, Kenya. \\ ${ }^{2}$ Mhadhiri Mwandamizi, Chuo Kikuu cha Karatina, S.L.P 1957-10101, Karatina, Kenya. \\ *Barua pepe ya mawasiliano: bensonsululu72@gmail.com.
}

DOI ya Nakala: https://doi.org/10.37284/eajss.3.1.373

\section{Tarehe ya Uchapishaji: IKISIRI}

02 Agosti 2021 Usomaji wa fasihi katika kipindi cha sasa unabainisha idadi kubwa ya waandishi kutoka jamii zisizo Waswahili ambao wameandika kazi katika tanzu

Istilahi Muhimu: mahsusi za fasihi simulizi ya Kiswahili. Waandishi hawa wameendelea

Waswahili, ka jamia maudhui, ploti na vipengele vingine vya utamaduni wa fasihi simulizi

Fasihi Simulizi, vya fasihi simulizi za jamii za waandishi hawa, wasiokuwa Waswahili,

Tanzu za Kimaigizo,

Riwaya ya Kiswahili, vimeingizwa katika riwaya ya Kiswahili na kuwa sehemu ya riwaya hii. Makala hii imechunguza jinsi uingizaji wa vipengele hivi vya fasihi simulizi

Umahuluti. hii kuwa ya kimahuluti. Mahsusi, makala hii imedadavua mchango wa tanzu za kimaigizo za fasihi simulizi katika umahuluti wa riwaya ya Kiswahili kupitia mfano wa riwaya ya Kufa Kuzikana (2003) iliyoandikwa na Ken Walibora. Tathmini hii imetumia Nadharia ya Umahuluti wa Utamaduni inayoshikilia kwamba panapotokea hali ya mtagusano baina ya tamaduni tofauti, matokeo yake si wingi-tamaduni bali ni mchanganyiko wa vipengele kutoka tamaduni hizi mbalimbali ambao ni bora na imara zaidi kuliko tamaduni asilia. Nadharia hii imetumiwa kufafanua jinsi umahuluti wa riwaya ya Kiswahili umechangiwa na uingizaji wa vipengele vya tanzu za kimaigizo za fasihi simulizi kutoka jamii za watunzi wake teule wasiokuwa Waswahili. Katika kufanya hivyo, makala hii imebainisha jinsi kuingizwa kwa vipengele hivi vya fasihi simulizi za jamii za waandishi teule katika riwaya ya Kiswahili kumeirutubisha riwaya hii kwa kuifanya kuwa changamano na nyumbufu kwa ambavyo inameza tanzu kutoka tamaduni tofauti na kujiimarisha kupitia kwazo bila kupoteza sura yake asilia.

91 This work is licensed under a Creative Commons Attribution 4.0 International License. 


\section{APA CITATION}

Sululu, S. B., Wafula, R. M., \& Maitaria, J. N. (2021). Mchango wa Tanzu za Kimaigizo za Fasihi Simulizi Katika Umahuluti wa Riwaya ya Kiswahili: Uchunguzi wa Riwaya ya Kufa Kuzikana. East African Journal of Swahili Studies, 3(1), 91-99. https://doi.org/10.37284/eajss.3.1.373.

\section{CHICAGO CITATION}

Sululu, Simiyu Benson., Richard Makhanu Wafula., and Joseph Nyehita Maitaria. 2021. "Mchango wa Tanzu za Kimaigizo za Fasihi Simulizi Katika Umahuluti wa Riwaya ya Kiswahili: Uchunguzi wa Riwaya ya Kufa Kuzikana". East African Journal of Swahili Studies 3 (1), 91-99. https://doi.org/10.37284/eajss.3.1.373.

\section{HARVARD CITATION}

Sululu, S. B. Wafula, R. M., and Maitaria, J. N. (2021) "Mchango wa Tanzu za Kimaigizo za Fasihi Simulizi Katika Umahuluti wa Riwaya ya Kiswahili: Uchunguzi wa Riwaya ya Kufa Kuzikana”, East African Journal of Swahili Studies, 3(1), pp. 91-99. doi: 10.37284/eajss.3.1.373.

\section{IEEE CITATION}

S. B. Sululu, R. M. Wafula, and J. N. Maitaria, "Mchango wa Tanzu za Kimaigizo za Fasihi Simulizi Katika Umahuluti wa Riwaya ya Kiswahili: Uchunguzi wa Riwaya ya Kufa Kuzikana”, EAJSS, vol. 3, no. 1, pp. 91-99, Aug. 2021.

\section{MLA CITATION}

Sululu, Simiyu Benson., Richard Makhanu Wafula., and Joseph Nyehita Maitaria.. "Mchango wa Tanzu za Kimaigizo za Fasihi Simulizi Katika Umahuluti wa Riwaya ya Kiswahili: Uchunguzi wa Riwaya ya Kufa Kuzikana”. East African Journal of Swahili Studies, Vol. 3, no. 1, Aug. 2021, pp. 91-99, doi:10.37284/eajss.3.1.373.

\section{UTANGULIZI}

Riwaya ni mojawapo ya tanzu ambapo waandishi wake wameibuka kutoka jamii tofauti za Kiafrika. Riwaya ya Kiswahili imeandikwa na waandishi ambao ni Waswahili na wasiokuwa Waswahili. Dhana 'Waswahili' katika muktadha wa makala hii inarejelea wazawa wa lahaja mojawapo za Waswahili na ambao wanatumia mojawapo ya lahaja za Kiswahili kama lugha yao asilia. Inafuatia basi kwamba, wapo watunzi wa riwaya ya Kiswahili ambao ni Waswahili na wengine, kama vile Ken Walibora, ambao si Waswahili. Asili ya Ken Walibora ni jamii ya Wabukusu. Licha ya kuwa asili ya mtunzi huyu si Waswahili, ameandika riwaya za Kiswahili. Riwaya ya Kiswahili katika muktadha huu ni riwaya ambayo utunzi wake unatilia maanani mikatale na mfumo wa lugha ya Kiswahili.

Waandishi wa riwaya ya Kiswahili, wasiokuwa Waswahili, hutumia tanzu mbalimbali za fasihi simulizi za jamii zao katika uandishi wa riwaya hii. Mojawapo ya tanzu hizo ni utanzu wa kimaigizo. Maigizo husheheni vitendo na msururu wa matukio yanayoibua hisia za washiriki (Maritim, 2012). Hata hivyo, matumizi ya lugha kisanaa katika maigizo haya ndiyo huyafanya kuwa ya kifasihi simulizi. Hii ina maana kwamba utendaji huenda sambamba na matumizi ya maneno hivi kwamba vipengele hivi viwili haviwezi kutenganishwa (Dundes, 1965). Hivyo basi, ni vigumu kutenganisha vipengele vya usanii wa lugha iliyotumiwa, na matukio ya kitamaduni kama vile ndoa, jando, mazishi na tohara.

Makala hii imeangazia tanzu za kimaigizo za fasihi simulizi katika muktadha wa mivigha ya kitamaduni kama inavyotekelezwa katika jamii ya Wabukusu, anakotoka Ken Walibora. Aidha, makala hii imetathmini jinsi mwandishi huyu alivyoingiza baadhi ya vipengele vya maigizo ya fasihi simulizi ya jamii yake katika riwaya teule ya Kiswahili na kuifanya kuwa ya kimahuluti.

\section{Tafiti Kuhusu Umahuluti}

Umahuluti ni zao la mtagusano wa kitamaduni, uhamisho na kubadilishana kwa vipengele kutoka tamaduni husika. Matokeo ya mchanganyiko huu wa tamaduni tofauti ni utamaduni ambao ni bora na imara zaidi (Bhabha, 1994). Kulingana na Rosaldo (1995) hakuna maeneo maalum yenye upekee kwa kuwa maeneo yote hupitia mfululizo wa mwingiliano wa tamaduni. Mwingiliano huu unahusisha kukopa na kupokeza vipengele fulani kati ya tamaduni tofauti. Kwa misingi hiyo, taaluma mbalimbali katika maisha ya binadamu, zikiwemo fasihi na sanaa kwa ujumla, zimeshirikisha dhana ya umahuluti kupitia njia ya mapokezano.

Tafiti mbalimbali zimefanywa kuhusu umahuluti. Kwa mfano, Ashcroft na wengine (2004) 
walichanganua namna ambavyo waandishi wa Kiafrika walioandika kazi za fasihi ya Kiingereza katika kipindi cha baada ya ukoloni walijumuisha vipengele vya utamaduni na lugha zao asilia katika tungo hizo. Wanataja mifano ya watunzi kama vile Gabriel Okara, Chinua Achebe na Ngugi wa Thiong'o ambao wametumia miundo ya kisintaksia, kisemantiki na kimofolojia kwa njia ambayo inakiuka sheria za sarufi ya Kiingereza. Tathmini hiyo inabainisha jinsi watunzi, wenye asili ya Kiafrika, walipigania kuhifadhi Uafrika katika riwaya za Kiingereza walizoandika.

Wafula (2008) alichunguza suala la umahuluti kupitia watunzi wa riwaya ya Kiswahili waliokopa mitindo ya kisanaa kutoka jamii za kigeni na kuijumuisha katika masimulizi ya riwaya zao za Kiswahili. Uchunguzi huo unaonyesha jinsi mtunzi kama Muhammad Said Abdulla alivyoiga mtindo wa mwandishi wa Kimagharibi Conan Doyle kwa kutumia mbinu ya upelelezi katika masimulizi yake. Aidha, Wafula (2008) alichunguza namna ambavyo waandishi wa riwaya ya Kiswahili kama vile Said Ahmed Mohammed na Kyalo Wamitila walivyochanganya uhalisia wa kiajabu wa masimulizi ya Amerika Kusini katika kuunda riwaya za Babu Alipofufuka (2001) na Bin-Adamu (2002) mtawalia. Vilevile, uchunguzi huo ulibainisha jinsi Shaaban Robert, katika riwaya ya Adili na Nduguze (1952), alivyokopa vipengele kama vile motifu kutoka hadithi ya 'Kisa cha Mbwa Wawili Weusi' inayopatikana katika mkusanyiko wa hadithi wa Alfu Lela Ulela (1928). Utafiti huo ulibainisha jinsi umahuluti unavyodhihirika katika riwaya ya Kiswahili kupitia mchango wa vipengele vya tamaduni za jamii za kigeni mathalan jamii za Amerika, na Uarabuni.

Okwena (2019) alichunguza umahuluti wa miundo katika tamthilia za Ebrahim Hussein. Lengo lake lilikuwa ni kuhakiki na kuchanganua matumizi ya miundo mbalimbali katika tamthilia tano za Ebrahim Hussein. Miundo hiyo ni ya ki-Aristotle ya tanzia, ya Brecht ya ki-utendi na fomyula za kisimulizi za masimulizi ya Kiafrika. Kwa hivyo, matumizi ya miundo anuwai ndilo suala lililochochea utafiti huo. Suala hilo lilichunguzwa kupitia tamthilia tano ambazo ni: Kinjeketile (1969), Mashetani (1971), Jogoo kijijini (1976), Ngao ya Jadi (1976) na Arusi (1980). Utafiti huo ulibainisha namna Ebrahim Hussein alivyoathiriwa na watunzi waliomtangulia kama vile Aristotle, Hegel na Brecht katika utunzi wa tamthilia. Aidha, utafiti huo ulibainisha namna elimu ya mtunzi Ebrahim Hussein kuhusu miundo waliyotumia watangulizi wake mbalimbali katika sanaa ya utunzi wa tamthilia ilivyochangia umahuluti wa miundo ya utunzi wa tamthilia zake mbalimbali.

Kwa ujumla, tafiti zilizopitiwa na mtafiti kuhusu umahuluti, zililenga umahuluti unaotokana na upya uliokopwa kutoka tamaduni na mazingira ya kigeni kama vile ya Uarabuni, Ulaya na Amerika na kuingizwa katika utunzi wa riwaya na tamthilia ya Kiswahili. Aidha, baadhi ya tafiti hizo zilishughulikia umahuluti unaotokana na kuingizwa kwa vipengele kutoka tamaduni za Kiafrika, na watunzi Waafrika, katika kazi andishi wanazoandika katika lugha za kigeni kama vile Kiingereza. Hata hivyo, katika ujumla wake, tafiti hizo zote hazikulenga kushughulikia umahuluti wa riwaya ya Kiswahili na namna umahuluti huo ulivyochangiwa na tanzu za fasihi simulizi za jamii za watunzi Waafrika, wasiokuwa Waswahili, wanaoandika riwaya ya Kiswahili.

\section{Mbinu za Utafiti}

Utafiti huu kuhusu mchango wa tanzu za fasihi simulizi katika umahuluti wa riwaya ya Kiswahili ulifanyiwa sehemu mbili: maktabani na nyanjani. Utafiti wa maktabani ulihusisha usomaji wa kina wa riwaya ya Kufa Kuzikana (2003) ili kupata data kuhusu matumizi ya tanzu za kimaigizo za fasihi simulizi, za jamii ya mtunzi teule, katika riwaya hii ya Kiswahili. Data kutoka katika kitabu kilichoteuliwa, ambayo ilihusisha maneno, sentensi, vishazi na vifungu vilivyo na ukuruba na maelezo kuhusu tanzu za kimaigizo za fasihi simulizi za watunzi teule, ilinakiliwa na kutumiwa hatimaye katika uchanganuzi. Vilevile, utafiti wa maktabani ulihusisha mapitio ya vyanzo mbalimbali mathalan vitabu, majarida na tasnifu ili kubainisha yaliyoandikwa kuhusu dhana ya umahuluti, utanzu wa riwaya, tanzu za fasihi simulizi na Nadharia ya Umahuluti wa Utamaduni.

Utafiti wa nyanjani ulimwezesha mtafiti kupata data ya msingi kuhusu tanzu za fasihi simulizi za jamii alikozaliwa na kukulia Ken Walibora ambaye riwaya yake ya Kiswahili ilichunguzwa katika utafiti huu. Utafiti wa nyanjani ulilenga kuwahoji 
wanajamii-lugha kutoka jamii ya Wabukusu anakotoka Ken Walibora. Data kutoka nyanjani ilipatikana kupitia matumizi ya mbinu za: maswali ya hojaji, mahojiano ya ana kwa ana na uchunzaji mahuluti. Hojaji ilitumiwa katika utafiti huu kwa mwandishi Ken Walibora ili kubainisha muktadha wa maisha ya mwandishi huyu unaoambatana na umahuluti wa vipengele vya mazingira tofauti alimoishi na kukulia. Kuhusu umuhimu wa hojaji kama mbinu ya ukusanyaji data, Kothari (2004: 101) anaeleza:

Hojaji huondoa hali ya mapendeleo kwa upande wa mtafiti kwa kuwa wahojiwa hutoa kauli zao kwa maneno yao wenyewe. Aidha, wahojiwa huwa na muda mwafaka wa kutoa majibu ambayo wameyatafakari kwa kina.

Kadhalika, mtafiti alitumia mbinu ya mahojiano ya ana kwa ana katika ukusanyaji wa data nyanjani. Kulingana na Mason (2002), mbinu ya mahojiano kama mbinu ya ukusanyaji data inahusisha mjadala kati ya mtafiti na mhojiwa ambapo mtafiti huuliza maswali ambayo hujibiwa na mhojiwa huku mtafiti akirekodi majibu. Kuhusu umuhimu wa mbinu ya mahojiano, Jwan na Ong'ondo (2011: 65-66) wanaeleza:

Mahojiano ni kati ya mbinu muhimu zaidi za kukusanya data katika utafiti wa kimaelezo kwa kuwa utafiti wa kimaelezo unahusu masuala yanayogusia mahusiano ya kijamii na mambo ambayo watu wanajua na kupitia katika maisha yao ya kila siku. Kwa hivyo, mahojiano huwapa wahojiwa fursa nzuri ya kueleza mambo hayo kwa kina na kwa uhakika zaidi.

Kupitia mbinu hii ya mahojiano ya ana kwa ana mtafiti alikusanya data iliyojumuisha masimulizi ya wahojiwa ya kipera cha embalu (tohara) cha utanzu wa kimaigizo wa fasihi simulizi ya jamii ya Wabukusu. Masimulizi haya yalinakiliwa na mtafiti katika maandishi na kurekodiwa katika kinasa sauti kwa minajili ya uchanganuzi. Uchanganuzi huo ulilenga kubainisha iwapo vipera vya utanzu wa kimaigizo wa fasihi simulizi ya jamii ya mtunzi teule, vilivyosimuliwa na wahojiwa, vilijibainisha katika riwaya ya Kufa Kuzikana (2003) na hivyo kuifanya riwaya hii ya Kiswahili kuwa ya kimahuluti.
Mbinu ya uchunzaji mahuluti ilitumiwa na mtafiti katika kukusanya data kuhusu kipera cha embalu (tohara) kama kinavyofanikishwa katika jamii ya Wabukusu. Wanjala (2020) anaeleza uchunzaji mahuluti kama mbinu inayojumuisha kushiriki, kushuhudia na kuhoji katika mchakato wa ukusanyaji wa data. Anafafanua kuwa mbinu hii hutumika zaidi katika utafiti unaohusu utamaduni wa watu au jamii kwa kuwa humwezesha mtafiti kukusanya data kuntu na hata ya siri na ambayo haiwezi kukusanywa na mbinu nyingine. Kwa hivyo, kupitia mbinu ya uchunzaji mahuluti mtafiti alitangamana moja kwa moja na wanajamii wa jamii ya Wabukusu katika sherehe za embalu (tohara) ili kubaini kwa kina hatua mbalimbali za utekelezaji wa kipera hiki. Data iliyokusanywa kupitia matumizi ya mbinu hii ilirekodiwa ili kutumiwa hatimaye katika uchanganuzi.

Data yote iliyokusanywa kwa minajili ya utafiti huu ilichanganuliwa ili kubainisha namna ambavyo uingizaji wa tanzu za kimaigizo za fasihi simulizi, za watunzi wasiokuwa Waswahili mathalan Ken Walibora, katika riwaya za Kiswahili walizoandika ulichangia umahuluti wa riwaya hizi. Katika hatua ya msingi, data kuhusu fasihi simulizi ya jamii ya mtunzi teule ambayo mtafiti alikusanya nyanjani ilinakiliwa na mtafiti mwenyewe. Kuhusu haja ya kunakili data mwenyewe, Jwan na Ong'ondo, (2011: 106) wanaeleza:

Inapendekezwa kwamba mtafiti anakili data mwenyewe bila usaidizi wa mtu mwingine. Kwa kufanya hivyo, mtafiti atapata fursa nzuri ya kufahamu data kwa misingi yake mbalimbali na hivyo kuielewa barabara.

Baada ya kuinakili, mtafiti aliipitia data hiyo mara kadha ili kuitathmini kwa misingi ya muundo wake, na uhusiano uliopo baina ya vipengele vyake mbalimbali. Kwa kuongozwa na usomaji wa data ya sekondari iliyojumuisha vitabu vya kihakiki ambavyo vilibainisha maana ya tanzu na vipera mbalimbali vya fasihi simulizi, mtafiti aliainisha data iliyojumuisha maelezo kuhusu ufanikishwaji wa kipera cha embalu (tohara). Baada ya kuiainisha, data hiyo ya nyanjani ilichanganuliwa kwa kuilinganisha na data kutoka katika riwaya ya $K u f a$ Kuzikana (2003) ili kudhihirisha iwapo kipera hicho cha utanzu wa kimaigizo wa fasihi simulizi kilijitokeza katika riwaya hiyo. Uchanganuzi huu 
ulifanywa kwa kuongozwa na lengo la utafiti huu ambalo ni kufafanua namna tanzu za kimaigizo za fasihi simulizi za watunzi teule zimechangia umahuluti wa riwaya ya Kiswahili, na mihimili ya Nadharia ya Umahuluti wa Utamaduni.

\section{Nadharia ya Umahuluti wa Utamaduni}

Tathmini hii iliongozwa na Nadharia ya Umahuluti wa Utamaduni. Nadharia hii inahusu fasihi na nadharia zinazotafiti na kuchanganua athari za mchanganyiko wa utambulisho wa utamaduni. Asili ya dhana umahuluti ni taaluma ya elimu-viumbe lakini baadaye ilijumuishwa katika utamaduni na isimu (Raab na Buttler, 2008). Nadharia ya Umahuluti wa Utamaduni, hivyo basi, ina misingi yake katika taaluma ya jenetiki ambayo ni kitengo cha elimu-viumbe. Kwa mujibu wa taaluma hii, unapochanganya jeni tofauti matokeo yake huwa bora kuliko unapochanganya jeni zinazofanana. Kwa misingi hiyo, mchanganyiko wa tamaduni tofauti huzaa utamaduni ambao ni bora na imara zaidi.

Bhabha (1994) ambaye ni mmojawapo wa waasisi wa nadharia hii, anaeleza umahuluti kama hali inayotokea wakati tamaduni mbili au zaidi zinapotagusana. Anafafanua kuwa, umahuluti ni dhana inayoashiria nafasi maalum ambapo mchakato wa mitagusano baina ya tamaduni mbalimbali hutokea. Naye Rosaldo (1995) anaeleza umahuluti kama hali inayoendelea daima katika tamaduni za binadamu wote. Ni hali ambayo inapinga kuwepo maeneo halisi yenye udhu kwa kuwa kila eneo hupitia mchakato huu wa mwingilianotamaduni unaohusisha ukopaji kutoka pande zote za tamaduni husika. Kwa hivyo umahuluti unatamalaki katika nyanja zote za maisha ya binadamu. Waitifaki wa nadharia hii kama vile Kraidy (2005) wanaeleza jinsi umahuluti ulivyokita mizizi katika jamii. Hivyo basi, taaluma mbalimbali katika maisha ya binadamu, zikiwemo fasihi na sanaa kwa ujumla, zimeshirikisha umahuluti kupitia njia ya mapokezano. Kwa hivyo, umahuluti ni dhana inayopinga hali ya uhalisi na kuwepo kwa namna moja ya vitu.

\section{Maigizo katika Muktadha wa Kifasihi Simulizi}

Licha ya kwamba maigizo husheheni vitendo na msururu wa matukio yanayoibua hisia za washiriki
(Maritim, 2012), matumizi ya lugha kisanaa katika maigizo haya ndiyo huyafanya kuwa ya kifasihi simulizi. Kwa mujibu wa Nandwa na Bukenya (1983), fasihi huelezwa kama matamko ambayo ama yamesemwa, yamekaririwa au kuimbwa. Haya ni matamko ambayo utunzi na utendaji wake, ambao huchukua mfumo wa kimaigizo, hudhihirisha kiwango kikubwa cha usanii. Hii ina maana kwamba utendaji huenda sambamba na matumizi maalum ya maneno hivi kwamba vipengele hivi viwili haviwezi kutenganishwa. Okpewho (1992) anaafiki hali hii anapoeleza kuwa fasihi simulizi ya kundi fulani la watu hujumuisha mambo mawili, yaani kile wanachosema watu hao na kile wanachotenda kwa mujibu wa utamaduni wao. Kauli hii inashadidiwa na Dundes (1965) ambaye anaeleza kwamba fasihi simulizi hupitishwa kutoka mtu mmoja hadi mwingine kwa njia ya maneno au matendo. Kwa hivyo, fasihi simulizi hufanikishwa katika mazingira asilia hivi kwamba ni vigumu kutenganisha vipengele vya usanii wa lugha iliyotumiwa na matukio ya kitamaduni yanayoambatana na maisha ya kila siku ya wanajamii.

\section{Miagizo katika Jamii ya Wabukusu}

Kama ilivyoelezwa na wahojiwa nyanjani, katika hatua mbalimbali za maisha yao Wabukusu hushiriki matendo ya kitamaduni yanayozingatia taratibu za kipekee. Matendo haya huchukua mfumo wa taratibu za kimatambiko zinazoeleweka na wanajamii. Maigizo ya kimatambiko huhusisha matendo ambayo hufungamana na hatua za mabadiliko anayopitia binadamu katika maisha yake kuanzia kuzaliwa hadi kufa (Were, 2014). Kila mojawapo ya hatua hizi huwa na umuhimu wa kipekee kwa kuwa huashiria mpito kutoka hatua moja hadi nyingine. Hatua hizi huathiri uhusiano wa mhusika na wanajamii wenzake kwa njia moja au nyingine. Miongoni mwa matendo ya kitamaduni yaliyotajwa na wahojiwa ni: embalu (tohara), khubeya (ndoa), lifwa nende lisikha (kifo na mazishi), bung'osi (utabiri), silamo (maapizo) na khukhwebula (kujitambulisha). Kila mojawapo ya matendo haya ya kimaigizo huhusisha taratibu zinazofuatwa kwa mujibu wa imani na maafikiano ambayo yamedumu na kuwa sehemu ya maisha ya Wabukusu. Baada ya kupitia riwaya ya Kufa Kuzikana (2003) mtafiti alibaini matumizi ya baadhi ya majukwaa haya ya kimaigizo na Ken Walibora 
katika uandishi wa riwaya hii. Hata hivyo, upeo wa utafiti huu utajikita katika ufafanuzi wa kina wa jukwaa moja: embalu (tohara). Jukwaa hili limefafanuliwa kwa kina ili kudhihirisha jinsi matumizi yake yalivyochangia umahuluti wa riwaya ya Kiswahili iliyoandikwa na Ken Walibora.

\section{Taratibu za Utekelezaji wa Embalu katika Jamii ya Wabukusu}

Embalu hufanyika kila mwaka shufwa katika jamii ya Wabukusu. Huhusisha mseto wa matendo na mazungumzo ambayo hufuata ruwaza maalum kuanzia mwanzo hadi kukamilika kwake. Kuhusu mseto huu wa matendo na matamko, Khamalwa (2004: 44) anaeleza:

Tambiko ni zaidi ya matendo ya kimwili kwa kuwa

huhusisha pia maneno yanayoyapa matendo hayo

uamilifu wake. Matambiko hayaigizwi tu bali pia

huzungumziwa. Huzungumziwa na wale wanaoyatekeleza.

Msimu wa embalu unapowadia, kila familia iliyo na kijana (omusinde) aliyetimu umri wa ada hiyo hutazamia utekelezwaji wake. Hata hivyo, shughuli yote huanza tu pale ambapo omusinde atajitokeza kwa hiari yake na kumfahamisha babake kuwa yu tayari kutahiriwa. Atamwendea babake na kumweleza azma yake. Kidesturi, babake, hata kama alikuwa akitazamia ujumbe huo, hastahili kukubali ombi la kijana moja kwa moja. Hujaribu kukataa na hata kutoa kauli zenye lengo la kuibadilisha azma yake ili kupima uthabiti wa kijana huyo. Babake omusinde akisharidhika kwamba azma ya kijana yake ni dhabiti, atampa kibali cha kuanza mchakato mzima wa embalu. Mchakato huo huhusisha kusafiri hadi maeneo mbalimbali kuwaalika jamaa zake kwenye sherehe ya tohara yake. Shughuli hiyo ambayo hujulikana kama khulanga huchukua siku kadhaa.

Baada ya kukamilisha shughuli ya khulanga hufuata hatua iitwayo khuchukhila ambayo huhusisha kitendo cha omusinde kwenda mtoni na chungu ambacho hukijaza maji na kurejea nacho nyumbani ambapo huyamwaga maji hayo kwenye mchanganyiko wa unga wa kutengenezea pombe uliokaangwa (kamakhalange) kama ishara kwamba hatobadili azma yake ya kutahiriwa. Hatua inayofuatia, ni ya kwenda kwa mjomba (ebwiwana) ambapo mjomba humchinja ng'ombe na kumvisha omusinde sehemu maalum ya ng'ombe huyo (luliki). Baada ya kuvishwa nyama hiyo ya luliki, omusinde na wale wanaoambatana naye wanapotoka kwa mjomba hufululiza moja kwa moja hadi nyumbani. Wanapofika nyumbani, ng'ombe mwingine (eye lisombo) huchinjwa na babake omusinde. Kisha, omusinde huvishwa kipande cha utumbo (lisombo) kutoka kwa ng'ombe huyo. Kipande hicho cha lisombo huwa ni ishara ya "nguo za mama' anazovaa mvulana katika umri wake wa utotoni kabla ya kutahiriwa. Katika jamii ya Wabukusu, mvulana yeyote asiyetahiriwa hurejelewa kwa jina 'mtoto' (omwana) hadi atakaposhiriki tambiko la tohara ambapo sasa atakuwa mwanamume (omusani). Kwa hivyo, anapovishwa nyama ya lisimbo ni ishara kwamba hiyo ni mara yake ya mwisho kuvaa nguo za mama au nguo za utotoni. Hicho kipande cha lisombo kitakuwa shingoni mwake hadi atakapotahirishwa asubuhi inayofuata baada ya kupelekwa mtoni (esitosi) kupakwa tope maalum (khulonga). Shughuli ya khulonga inapokamilika, mwanamume mmoja huongoza uimbaji wa wimbo maalum (sioyayo) kisha safari ya kurejea nyumbani huanza. Uimbaji wa sioyayo huashiria kilele cha shughuli ya embalu. Omusinde huanza safari ya kurejea nyumbani kukabiliana na kisu cha ngariba.

Shughuli ya kutahirisha inapokamilika vizuri kijana huyo hupewa zawadi za kila aina kwa ujasiri wake kisha hufunikwa blanketi au leso. Kufunikwa huko huashiria kwamba watoto na wanawake walioko watakuwa wameuona uchi wake kwa mara ya mwisho. Yeye atakuwa mwanamume sasa na wala si mtoto tena. Mara tu baada ya kutahiriwa na kwa kipindi ambapo atakuwa akiuguza jeraha lilitokana na upashwaji tohara, ataitwa omufulu (anayeuguza jeraha). Baada ya kupona jeraha, atafanyiwa sherehe maalum iitwayo sisialukho ambapo hupewa wasia katika sherehe iitwayo khubita. Sherehe hiyo ndiyo huashiria mwisho wa mchakato wa embalu. Kijana akishafanyiwa lubito, atakuwa amekamilisha safari ya kutoka utotoni na kuingia utu uzima. Sasa atakuwa huru kushiriki mambo yote wanayoshiriki wanaume kama vile kuoa, na kuwa miongoni mwa jeshi la kuilinda jamii yake. Daima atakuwa na uhusiano wa kipekee na wenzake wa 
rika moja ambao walitahiriwa msimu mmoja (bakoki).

Hata hivyo, pakitokea hali ambako kijana aliyefikisha umri wa kutahiriwa atakosa kuonyesha ishara yoyote kwamba yu tayari kwa ada hiyo, itabidi wanajamii waingilie kati. Aghalabu mtu kama huyo hupashwa tohara ya lazima. Mwanajamii anayetahirishwa kwa lazima huubeba mzigo wa fedheha na kukejeliwa na wanajamii wenzake maishani mwake mwote. Nao vijana wa kiume waliokimbilia maeneo ya mbali ili kukwepa kisu cha ngariba husakwa kule waliko na kutahirishwa kwa lazima.

\section{Mchango wa Baadhi ya Vipengele vya Embalu katika Umahuluti wa Riwaya ya Kufa Kuzikana}

Kama mzawa wa jamii ya Wabukusu, Ken Walibora, katika uandishi wa riwaya ya Kufa Kuzikana (2003), anaelekea kuathiriwa na fasihi simulizi ya jamii yake anakozaliwa. Anaposimulia maisha ya wahusika wake, anatumia baadhi ya matukio ambayo yanaambatana na uhalisia wa jinsi matukio hayo yanavyofanikishwa katika jamii wa Wabukusu. Kama ilivyo katika jamii ya Wabukusu ambapo jukwaa la embalu huwa na umuhimu mkubwa kwa mwanajamii hasa wa jinsia ya kiume, Walibora anatalii suala hili na kulipa umuhimu mkubwa kupitia baadhi ya wahusika wake katika riwaya yake ya Kufa Kuzikana (2003). Katika riwaya hii, mhusika Akida Sululu anasimulia tukio aliloshuhudia jijini Tandika lililohusisha tohara ya lazima. Tukio hilo linaenda sambamba na namna wafanyavyo Wabukusu wakati mmoja wa wanajamii anapokaidi kutimiza ada ya tohara kama inavyohitajika na jamii. Mhusika Akida anaeleza jinsi alivyoona umati mkubwa wa watu, wanawake kwa wanaume, waliopamba barabara, shughuli yao kuu ikiwa kumpeleka 'mtoto' hospitalini ili apashwe tohara ambayo alikuwa ameikimbia kwao. Aliyerejelewa kama 'mtoto' alikuwa mwanamume mkomavu kabisa wa umri wa zaidi ya miaka thelathini. Mhusika Akida alielezwa habari za 'mtoto' huyu na mpita njia mmoja aliyesema:

Huyu bwana tukibeba naye mizigo soko mjinga kwa miaka mingi. Tangu hapo hatujui mwenzetu kakimbia kisu cha ngariba. Ni Mchungwachungwa huyu jamani na tohara ni jambo la lazima kwa wanaume wote miongoni mwetu sisi Wachungwachungwa (uk.55).

Dhana hii ya mtu mkomavu wa jinsia ya kiume kuitwa 'mtoto' inatokana na yaliyoelezwa na wahojiwa kuambatana na mchakato wa utekelezwaji wa embalu. Kama ilivyoelezwa na wahojiwa, katika jamii ya Wabukusu mtu yeyote wa kiume asiyepashwa tohara hurejelewa kama 'mtoto'. Huyu ni mtu anayesemekana kuvalia nguo za utotoni. Wakati wa zoezi la embalu, omusinde anapotoka kwa mjomba yake na kufika bomani kwa baba yake, huchinjiwa ng'ombe (eye lisombo) ambapo sehemu ya utumbo kutoka kwa ng'ombe huyo huvishwa shingoni mwake hadi atakapopashwa tohara siku inayofuatia. Utumbo huo ni ishara ya nguo za utotoni ambazo huvishwa kwa mara ya mwisho kabla ya kuvuka hadi kundi la utu uzima pale anapotahirishwa. Mara tu ngariba anapokamilisha zoezi la tohara, kijana huvuliwa utumbo huo kama ishara ya kuvuliwa nguo za utotoni na kufunikwa blanketi au shuka inayoashiria hatua mpya (utu uzima) katika maisha yake.

Kwa hivyo, Ken Walibora anarejelea baadhi ya hatua katika utekelezaji wa utamaduni wa miviga ya kimaigizo ya embalu anapozungumzia dhana hii ya 'mtoto'. Kwamba, miongoni mwa Wabukusu dhana ya 'mtoto' haikadiriwi kwa kipimo cha umri bali kwa kigezo cha iwapo mhusika amepashwa tohara au la. Hatua wanazochukua wanajamii wa jumuiya hii dhidi ya huyu 'mtoto' zinatokana na sababu kwamba ipo misururu ya mambo yanayofungamana na kipera cha utanzu wa kimaigizo cha embalu ambayo alihitajika kuwa ameipitia katika umri wake huo. Hata hivyo, kwa kuikaidi ikabidi wanajamii hao wachukue hatua waliyochukua ambayo pia inatambuliwa kama suluhu kwa hali kama hizo katika jamii yao. Maamuzi hayo wanayochukua wanajamii yanakwenda sambamba na hali jinsi ilivyo katika jamii ya Wabukusu kama alivyosimuliwa mtafiti na wahojiwa.

Kadhalika, mhusika Akida naye anapokimbilia usalama eneo la Sangura, anajipata miongoni mwa jamii ambapo tohara ilikuwa ni lazima, sawia na ilivyo katika jamii ya Wabukusu. Akida alikuwa hajatahiriwa na siri yake iligunduliwa na Cynthia, bintiye Mzee Muyaka, ambaye alimfumania akienda haja ndogo kwenye uchochoro mmoja katika kituo cha biashara cha Binge. Baadaye 
Cynthia anamtahadharisha Akida kuhusu hali yake anapomwambia:

Sasa yakupasa Mchungwachungwa uchunge sana. Watu wa Sangura wakijua hali yako utatiwa suna kwa nguvu. Wanawake wa Sangura wanatahiriwa sembuse wanaume (uk. 150).

Taswira anayochora mwandishi inakwenda sambamba na hali jinsi ilivyo katika jamii ya Wabukusu ambapo ni lazima kwa vijana wote wa kiume kupitia ada ya kimaigizo ya embalu.

Usawiri wa matendo yanayofungamana na miviga ya kimaigizo ya embalu na Ken Walibora katika riwaya ya Kufa Kuzikana (2003) unaonyesha jinsi vipengele vya fasihi simulizi ya wanajamii wa jamii ya Wabukusu, anamotoka mwandishi huyu, vimepenyezwa katika uandishi wake. Katika jamii hii ya Wabukusu tohara ni ada ya lazima kwa wavulana wote. Ni daraja la kuadhimisha kipindi anachopitia kila mwanajamii wa jinsia ya kiume katika safari yake ya kuingia utu uzima kama walivyoeleza wahojiwa. Ken Walibora, katika riwaya yake teule, anasawiri safari hii kwa kutumia baadhi ya matukio ambayo yanafungamana na utekelezaji wa embalu. Kwa njia hiyo anafanikisha riwaya yake kimuundo kwa kutumia usimulizi unaosheheni utajiri wa matendo ya maigizo ambayo yanalandana na yale ya fasihi simulizi ya jamii yake.

Hali hii inaonyesha mchango wa tanzu za kimaigizo za fasihi simulizi ya jamii ya Wabukusu katika uandishi wa riwaya teule ya Kiswahili ya Ken Walibora. Kama ilivyobainishwa kupitia Nadharia ya Umahuluti wa Utamaduni, mwandishi huyu anadhihirisha kwamba panapokuwepo na mwingiliano wa mifumo mbalimbali ya tamaduni, hutokea uhamisho na kubadilishana kwa mambo yanayohusu jadi, maadhimisho na miviga baina ya jamii ya mtunzi na jamii ya lugha pokezi anayoitumia kufanikisha kazi yake ya sanaa. Hali hii ndiyo inayochangia umahuluti wa riwaya ya Kiswahili kama inavyodhihirishwa kupitia mfano wa riwaya iliyoandikwa na Ken Walibora.

\section{Hitimisho}

Kwa ujumla, makala hii imedhihirisha jinsi watunzi wa riwaya ya Kiswahili wasiokuwa Waswahili, kama vile Ken Walibora, wametumia baadhi ya vipengele vya fasihi simulizi za jamii zao katika utunzi wao. Ken Walibora, ambaye ni mzaliwa wa jamii ya Wabukusu, ametumia baadhi ya tanzu za fasihi simulizi za jamii yake katika riwaya ya Kiswahili ya Kufa Kuzikana (2003). Katika riwaya hii, mwandishi ametumia baadhi ya vipengele vinavyofungamana na utekelezaji wa kipera cha kimaigizo cha embalu, cha jamii ya Wabukusu, katika usimulizi wa matukio. Kupitia kipera hiki, mwandishi ameweza kujenga msingi wa ufanikishaji wa maudhui na usawiri wa wahusika katika riwaya hii. Mathalan, mwandishi anaeleza jinsi mhusika kwa jina Akida aliuona umati mkubwa wa watu waliopita katika mitaa mbalimbali jijini Tandika wakiwa na 'mtoto' ambaye alipaswa kupashwa tohara ya lazima. Vitendo vilivyoambatana na tukio hilo vina ukuruba na ufanikishwaji wa miviga ya kimaigizo ya embalu miongoni mwa Wabukusu ambapo tohara ni lazima kwa vijana wote waliotimu umri na mtu yeyote wa jinsia ya kiume asiyepashwa tohara hurejelewa kama mtoto licha ya umri wake. Nadharia ya Umahuluti wa Utamaduni, ambayo imehimiliwa kwenye dhana ya kubadilishana kwa vipengele kutoka tamaduni tofauti, imetumiwa kufafanua namna uingizaji wa vipengele vya fasihi simulizi ya jamii ya Ken Walibora katika riwaya ya Kiswahili ya Kufa Kuzikana (2003) kumechangia umahuluti wa riwaya hii na riwaya ya Kiswahili kwa ujumla.

\section{MAREJELEO}

Ashcroft, B., Giffiths, G. \& Tiffin, H. (2004). The Empire Writes Back: Theory and Practice in Post-Colonial Literatures. ( $2^{\text {nd }}$ Edition). New York: Routledge.

Bhabha, H. K. (1994). The Location of Culture. New York: Routledge.

Dundes, A. (1965). The Study of Folklore. Englewood Cliffs, N. J: Prentice-Hall, Inc.

Jwan, J. O. \& Ong'ondo, C. O. (2011). Qualitative Research: An Introduction to Principles and Techniques. Eldoret: Moi University Press.

Khamalwa, J. P. W. (2004). Identity, Power, and Culture: Imbalu Initiation Ritual Among the Bamasaba of Uganda. Bayreuth: Bayreuth African Studies Series. 
Kothari, C. R. (2004). Research Methodology: Methods and Techniques ( $2^{\text {nd }}$ Revised Edition). New Delhi: New Age International (P) Limited, Publishers.

Kraidy, M. (2005). Hybridity or the Cultural Logic of Globalization. Philadelphia: Temple.

Maritim, E. (2012). 'The Concept and Nature of Drama and Theatre in Traditional African Societies.' in: African Drama \& Theatre A Criticism. Nairobi: Focus Publishers Ltd.

Mason, J. (2002). Qualitative Research $\left(2^{\text {nd }}\right.$ Edition $)$. London: SAGE Publications.

Mohamed, S. A. (2001). Babu Alipofufuka. Nairobi: Jomo Kenyatta Foundation.

Nandwa, J. \& Bukenya, A. (1983). African Oral Literature for Schools. Nairobi: Longman Kenya.

Okpewho, I. (1992). African Oral Literature: Backgrounds, Character, and Continuity. Bloomington, IN: Indiana University Press.

Okwena, S. (2019). "Umahuluti wa Miundo katika Tamthilia za Ibrahim Hussein". Tasnifu ya Uzamifu, Chuo Kikuu cha Kenyatta. (Haijachapishwa).

Raab, J. \& Buttler, M. (2008). Hybrid Americas: Contracts, Contrasts and Conferences in New World Literatures and Cultures. Verlag. Tempe: Bilingual Press.

Robert, S. (1952). Adili na Nduguze. London \& Basingtoke: Macmillan Publishers.

Rosaldo, R. (1995). "Foreword" in: Hybrid Cultures: Strategies for Entering and Leaving Modernity (Nestor Garcia Canclini). Tafsiri: Chiappari, C.L \& Lopez, S.L. Minneapolis: University of Minnesota Press.

Wafula, R. M. (2008). 'Performing Identity in Kiswahili Literature' in: Culture, Performance \& Identity (ed. Kimani Njogu). Nairobi: Twaweza Communications.

Walibora, K. (2003). Kufa Kuzikana. Nairobi: Longhorn Publishers (Kenya) Ltd.
Wamitila, K. W. (2002). Bin-Adamu. Nairobi: Phoenix Publishers.

Wanjala, F. S. (2020). Safina ya Utafiti wa Fasihi Simulizi. Kakamega: Elgon Epitome Publishers Limited.

Were, W. (2014). “A Traditional Ritual Ceremony as Edurama: A Case Study of Imbalu Ritual Among the Babukusu of Western Kenya". PhD Thesis, Kenyatta University. (Unpublished).

99 | This work is licensed under a Creative Commons Attribution 4.0 International License. 